\title{
A Method of Correcting for Tilt from Horizontal in Downwelling Shortwave Irradiance Measurements on Moving Platforms
}

\author{
${ }^{I}$ Pacific Northwest National Laboratory, Richland, WA, USA \\ ${ }^{2}$ Naval Research Laboratory, Monterey, CA, USA \\ ${ }^{3}$ NPS/CIRPAS, Marina, CA, USA \\ ${ }^{4}$ Brookhaven National Laboratory, Upton, NY, USA \\ ${ }^{5}$ Peak Design Ltd, Winster, Derbys, UK
}

C.N. Long ${ }^{*}, 1$, A. Bucholtz ${ }^{2}$, H. Jonsson ${ }^{3}$, B. Schmid ${ }^{1}$, A. Vogelmann ${ }^{4}$ and J. Wood ${ }^{5}$

\begin{abstract}
The downwelling shortwave irradiance typically consists of both a direct component of radiation from the sun, and a diffuse component of scattered sunlight from the sky. Significant offsets can occur in downwelling shortwave irradiance measurements made from moving platforms due to the tilt of the instruments from horizontal which changes the angular orientation of the direct component of sunlight to the instrument and causes an artificial variation in the measured signal. To properly correct for this tilt, a-priori knowledge of the partitioning between the direct and diffuse components of the total shortwave irradiance is needed to properly apply a correction for tilt. This partitioning information can be adequately provided using a newly available commercial radiometer named the SPN1 that produces reasonable measurements of the total and diffuse shortwave irradiance (and by subtraction the direct shortwave irradiance) with no moving parts and regardless of azimuthal orientation. We have developed methodologies for determining the constant pitch and roll offsets of the radiometers for aircraft applications, and for applying a tilt correction to the total shortwave irradiance data. Results suggest that the methodology is accurate for tilt up to $+/-10^{\circ}$, with $90 \%$ of the data corrected to within $10 \mathrm{Wm}^{-2}$ at least for clear-sky data. Without a proper tilt correction, even data limited to $5^{\circ}$ of tilt can still exhibit large errors, greater than $100 \mathrm{Wm}^{-2}$ in some cases. Given the low cost, low weight, and low power consumption of the SPN1 total and diffuse radiometer, opportunities previously excluded for moving platform measurements such as small Unmanned Aerial Vehicles and solar powered buoys now become feasible using our methodology. The increase in measurement accuracy is important, given current concerns over long-term climate variability and change especially over the $70 \%$ of the Earth's surface covered by ocean where long-term records of these measurements are sorely needed and must be made on ships and buoys.
\end{abstract}

Keywords: Tilt correction, shortwave irradiance, solar radiation, aircraft radiation measurements.

\section{INTRODUCTION}

A long-standing problem in making accurate downwelling shortwave (SW) irradiance measurements from aircraft and other moving platforms has been the effect of tilt from level of the radiometer detectors due to the motion of the platform. Non-level orientation can have a significant impact because, when the sun is not completely blocked by overhead cloud, the downwelling SW consists of both a prominent direct component of radiation from the sun, and a diffuse component of scattered sunlight from the sky, and any tilt of the instruments will change the angular orientation of the direct component of sunlight to the sensor and cause an artificial variation in the signal. One possible solution is the use of actively stabilized platforms, such as that described in Bucholtz (JTech, 2008) [1] and Wendisch (JTech, 2001) [2], which greatly decreases the measurement uncertainties due to the pitch and roll of the aircraft. However, while stabilized platforms are clearly the way to

*Address correspondence to this author at the Pacific Northwest National Laboratory, Richland, WA, USA; Tel: 1 (509) 372-4917; Fax: 1 (509) 3726247; E-mail: chuck.long@pnl.gov make the most accurate solar irradiance measurements from an aircraft they are still an emerging technology. They are complex and expensive to develop and maintain, and therefore are not widely available.

Previous attempts to correct SW irradiances for tilt have had limited success primarily because only the direct component of the total SW should have a tilt correction applied (the diffuse, more isotropic SW component being far less affected) and thus, a-priori knowledge of the partitioning of the total downwelling SW between the direct and diffuse components is required in order to accurately apply a tilt correction. Without knowledge of the direct and diffuse partitioning, one choice is to apply a "full correction" which assumes that all the downwelling solar radiation comes from the direct sun. As we will show, this approach can still leave relatively large errors in the resultant values. Other researchers have attempted to estimate the directdiffuse ratio by using climatology or radiative transfer models (e.g. Bannehar, NCAR Tech, 1991 [3]; Saunders, Jtech, 1992 [4]; Boers, JGR,1998 [5]; McDowall, MS FSU, 2005 [6]), but these methods have difficulty in varying aerosol and cloud conditions. In any case, not having either a 
stabilized platform or a-priori knowledge of the direct and diffuse partitioning, often the "rule of thumb" practice has been to only accept measurements with tilt from horizontal of $5^{\circ}$ or less as "usable" data to attempt to correct (see discussion in [1]).

A new commercial radiometer has recently become available that produces reasonable measurements of the total and diffuse, and by subtraction the direct, SW with no moving parts and regardless of azimuthal orientation. This radiometer is relatively light weight with modest power requirements, and was flown during a long-term aircraft field experiment as part of the suite of on-board radiometers. Using the measurements of the direct and diffuse partitioning, we have developed a methodology for correcting for tilt from horizontal in the downwelling total SW aircraft data. This tilt correction methodology, as we will show, appears to be highly successful for tilt up to $10^{\circ}$, and thus affords the opportunity for more accurate SW measurements for a range of moving platform applications that have been precluded to date.

\section{DATA}

As an integral measurement capability of the Atmospheric Radiation Measurement (ARM) Climate Research Facility (ACRF), the ARM Aerial Facility (AAF) provides airborne measurements required to answer science questions proposed by the ARM Science Team and the external research community. The AAF enhances the utility of long-term ground-based measurements by providing sampling not possible from the ground to provide context for and extend the ground-based measurements. The AAF supports intensive field campaigns or long-term, regularlyscheduled flights to acquire airborne measurements using the appropriate aerial vehicle and instruments. Aircraft choice is dictated by science requirements such as the required measurements, desired flight profile, and aircraft availability. AAF has access to a broad array of aircraft to address the wide range of aircraft measurement requirements associated with atmospheric science issues. Data obtained from the aircraft are documented, checked for quality, integrated into the ARM Data Archive, and made freely available for use by the scientific community. Recent AAF field campaigns include Cloud and Land Surface Interaction Campaign (CLASIC, 2007), Indirect and Semi-Direct Aerosol Campaign (ISDAC, 2008), and the Routine AAF Clouds with Low Optical Water Depths (CLOWD) Optical Radiative Observations (RACORO, 2009) discussed here. From 2007-2009, the ARM Aerial Facility was known as the ARM Aerial Vehicles Program, which was the successor to the 1993-2006 ARM Unmanned Aerospace Vehicle (UAV) Program.

The RACORO field campaign (Vogelmann, ARM Tech 2008) [7] was conducted from 22 January to 30 June, 2009 above the ACRF Southern Great Plains (SGP) site near Lamont, Oklahoma. The purpose of the campaign was to obtain representative statistics of boundary layer (water) clouds that are needed to support process studies, model simulations, and validate retrieval algorithms. By nature, the clouds targeted are optically thin, often occur in partly cloudy skies, and thus often include some direct SW and a significant diffuse component. This is the first time that a long-term aircraft campaign was undertaken for systematic in-situ sampling of cloud field properties and, over the fivemonth period, the RACORO team logged an unprecedented 59 flights and 259 research hours. Coordinated by the AAF, the Center for Interdisciplinary Remotely Piloted Aircraft Studies (CIRPAS) Twin Otter aircraft was equipped with a full payload of research instrumentation to obtain representative statistics of cloud microphysical, aerosol, and radiative properties of the atmosphere. Owing to the longterm nature of the program, the aircraft payload was kept as light and simple as possible to enable the cost effectiveness needed for routine observations. The currently available stabilized platform from Bucholtz [1] used over SGP in 2003 (Guan, JGR 2009) [8] was incompatible with the RACORO payload because its weight and power consumption would have limited the number of other instruments that could have been flown and the platform is not yet ready for long term, turnkey operations.

\section{MEASUREMENTS}

\section{Modified CM-22 Pyranometer}

A modified Kipp \& Zonen CM-22 pyranometer (Bucholtz, JTech in preparation [9]; Kipp \& Zonen, 2003 [10]) was mounted on top of the CIRPAS Twin Otter aircraft for RACORO to measure the downwelling broadband solar (0.2-3.6 $\mu \mathrm{m})$ irradiance (Fig. 1). The modified CM-22 retains the fundamental components of the original instrument, which was primarily designed for surface-based measurements, and incorporates changes to make it better suited for aircraft-based measurements. The modifications include a new back housing that retains the front end optics and electronics of the original instrument but allows an amplifier to be mounted directly below the sensor. With the signal amplified right at the sensor the instrument is run in current loop mode, a well established technique for minimizing the effects of noise in long signal cables. This technique is especially effective in the electronically noisy environment of a research aircraft. The new housing also has the cable connector on the bottom of the instrument for easier mounting on the aircraft. Even before modification the CM-22 pyranometer has features that make it attractive for aircraft use. For example, it utilizes electrical compensation circuitry, a quartz dome with high thermal conductivity, and good thermal coupling of the dome to the body to minimize the effects of temperature on the measured signal [10]. The temperature dependency of the sensitivity of the CM-22 was measured by Kipp \& Zonen and found to stay within approximately $+0.2 \%$ to $-0.3 \%$ over the $-20 \mathrm{C}$ to $+50 \mathrm{C}$ temperature range.

The sensitivity of the modified CM-22 was calibrated inhouse by comparison to a reference standard pyrheliometer using the alternating sun-shade method (ASTM, 2005 [11]). The reference pyrheliometer is an Eppley Model AHF SelfCalibrating Cavity Pyrheliometer (Hickey, Proc ASES, 1993 [12]) that participated in the National Renewable Energy Lab Pyrheliometer Comparison in October 2008 in Golden, Colorado, There it was compared to absolute pyrheliometers from the World Radiometric Group (WRG) and is therefore traceable to the World Radiometric Reference (WRR). The absolute uncertainty of the modified CM-22 is estimated to be $1-3 \%$ while the precision is estimated to be $1 \%$. A recent 
study by Guan [8] compared aircraft measurements of the downwelling solar irradiance from a similarly modified CM22 with model generated values for a variety of aerosolloading conditions and found that the measurements and model values agreed to within $0.8 \%$.

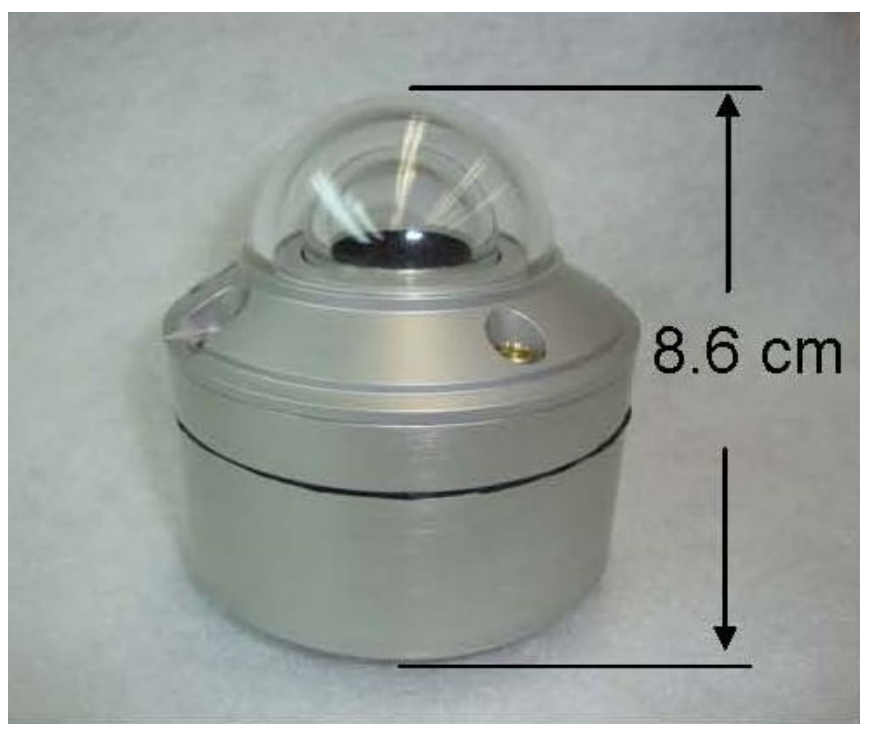

Fig. (1). Side view of the modified Kipp and Zonen CM-22 radiometer used for RACORO.

\section{SPN1 Total/Diffuse Radiometer}

The model SPN1 (sold by Delta-T Devices, UK) is a novel solar radiation sensor which can measure the direct and diffuse components of solar radiation without any moving parts or any specific alignment (Fig. 2). Thus this radiometer can work effectively on moving platforms such as aircraft, buoys, or ships. The SPN1 consists of an array of 7 radiation sensors in a hexagonal pattern, underneath a hemispherical shadowmask which has areas cut away, such that for any position of the sun in the sky:

- At least one sensor is always exposed to the direct solar beam

- At least one sensor is always completely shaded from the direct beam

- All sensors receive about equal amounts of diffuse light from the sky hemisphere

The pattern of cutaways in the hemispherical shadowmask was generated by computer using an evolutionary algorithm so that, from the viewpoint of each of the 7 sensors, the proportion of diffuse light received is very close to $50 \%$ for both a Uniform Overcast Sky, and a Standard Overcast Sky (Moon, Proc TIES, 1943 [13]). The images in Fig. (3) show the parts of the sky hemisphere "seen" by the different sensors as plotted using an equiangular projection, where radial distance on the image represents zenith angle on the hemisphere. The three different sensor "view" classes are shown, but specific sensors may actually see reflections of these in the horizontal or vertical plane. For example, sensor 2 "sees" the middle pattern as shown, while sensor 5 "sees" the horizontal mirror of this pattern.

As noted above, at any particular time there will always be one or more sensors which are fully shaded from the
Direct beam, but exposed to about half of the Diffuse radiation, several sensors which are partly exposed, and one or more which are fully exposed to the Direct beam plus about half of the Diffuse radiation. The seven sensors are measured in quick succession. The one with the lowest reading (MIN) is the one exposed to only $50 \%$ Diffuse. The one with the highest reading (MAX) is the one exposed to Direct plus 50\% Diffuse. The Direct and Diffuse can therefore be calculated as follows:

$$
\begin{aligned}
& \text { Diffuse }=2 * \text { MIN } \\
& \text { Direct }=\text { MAX }- \text { MIN } \\
& \text { Total }=\text { Direct }+ \text { Diffuse }=\text { MAX }+ \text { MIN }
\end{aligned}
$$

The calculated Total and Diffuse values are used for the instrument output.

There are two main sources of error specific to this design, in addition to the usual errors due to imperfections in calibration, cosine response and spectral response common to all radiation sensors: Sensor matching and diffuse sky sampling. The operation of the sensor relies on close matching between all seven sensors at all zenith and azimuth angles. There is typically a variation of $1 \%-2 \%$ between sensors due to manufacturing tolerances, and this can show up as a variation in the output as different sensors move from shaded to exposed. As the sensor sees only a $50 \%$ sample of the diffuse sky, variations in diffuse intensity on a scale similar to that of the shading pattern will also show up as variation in the instrument output.
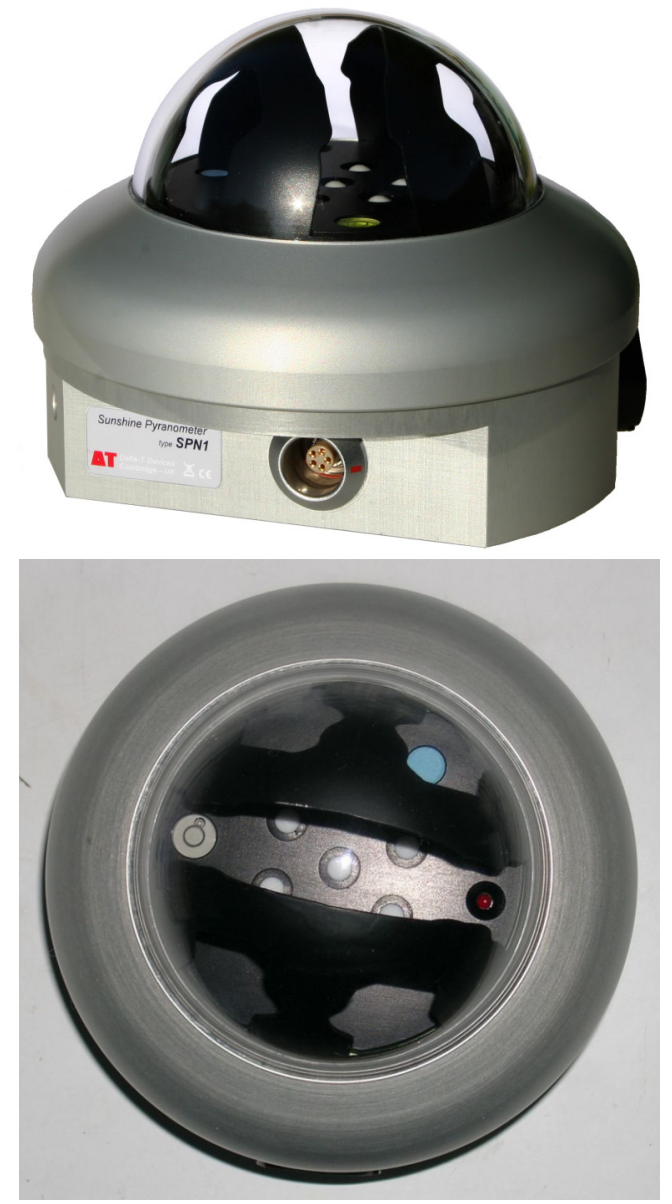

Fig. (2). Side and top views of the SPN1 radiometer. 


\section{Aircraft NAV Data}

For the RACORO experiment, the aircraft navigation data were provided by a Systron Donner Inertial C-Migits III GPS-based nav system (http://www.systron.com). Manufacturer specifications list pitch and roll 1-sigma accuracy as $1.0 \mathrm{mrad}$, and for heading as $1.5 \mathrm{mrad}$. The 1sigma accuracy for position is about $4 \mathrm{~m}$. These data were sampled at $10 \mathrm{~Hz}$ during the RACORO flights, and thus while the radiometer data were sampled at a higher rate (100 $\mathrm{Hz}$ ), the need for tilt correction using the nav data restricted the radiation data to $10 \mathrm{~Hz}$ averages as well.

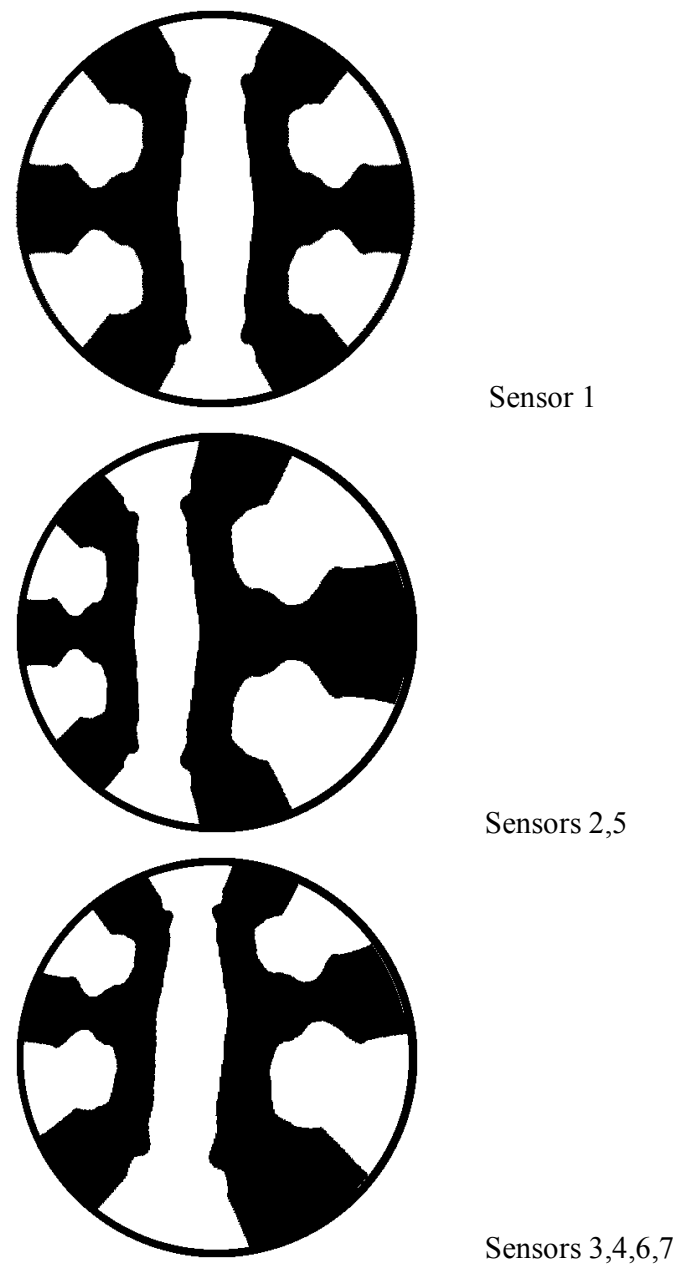

Fig. (3). Equiangular projection of the sky view of the three different sensor classes (by position) of the SPN1 radiometer.

\section{TILT CORRECTION METHODOLOGY}

The total downwelling SW irradiance, typically given in $\mathrm{Wm}^{-2}$, can be expressed as the sum of the direct and diffuse SW components:

$\mathrm{G}=\mathrm{D}+\mathrm{R}=\mathrm{D}+\mathrm{N}^{*} \mu_{0}$

where:

$\mathrm{G}=$ the total (global) downwelling SW

$\mathrm{D}=$ the downwelling diffuse $\mathrm{SW}$

$\mathrm{R}=$ the downwelling direct component $\mathrm{SW}$

$\mathrm{N}=$ the direct normal SW, i.e. that measured by a detector perpendicular to the sun's rays $\mu_{0}=$ the cosine of the solar zenith angle

This measurement assumes that the total SW instrument detector is horizontally oriented, i.e. parallel to the (flat) Earth surface. For a detector that is tilted from the horizontal, the similar formula would be:

$\mathrm{G}_{\mathrm{T}}=\mathrm{D}_{\mathrm{T}}+\mathrm{N}^{*} \mu_{\mathrm{T}}$

where the subscript " $\mathrm{T}$ " stands for the tilted case and $\mu_{\mathrm{T}}$ denotes the cosine of the angular relationship between the location of the sun in the instrument field-of-view and the normal to the plane of the detector. Note that in both cases, the direct normal value " $\mathrm{N}$ " is the same, since that quantity has a fixed orientation pointed toward the sun.

The purpose of applying a tilt correction is to modify the measured SW value $G_{T}$ such that it well represents the horizontally oriented value G. Combining Equations 1 and 2, and using three two-dimension coordinate transformations (matrix transform techniques are well known and thus not discussed here), the formula for correcting for tilt from horizontal becomes:

$$
G=G_{T}\left(\frac{\mu_{0}+D / N}{\mu_{T}+D_{T} / N}\right)
$$

Since the diffuse SW is by nature aptly named (i.e. it is to first approximation a diffuse, isotropic field measurement) the value of the diffuse SW should not be overly affected by modest tilt of the detector. Thus in the modest tilt case we assume that $\mathrm{D} \approx \mathrm{D}_{\mathrm{T}}$ and Equation 3 becomes:

$$
G=G_{T}\left(\frac{\mu_{0}+K}{\mu_{T}+K}\right)
$$

where $\mathrm{K}=\mathrm{D} / \mathrm{N}$, i.e. the diffuse to direct normal ratio.

Using navigation data for information for the latitude, longitude, pitch, roll, heading, date, and time one can calculate $\mu_{0}$ and $\mu_{\mathrm{T}}$. The problem has always been gaining knowledge of the partitioning of the total SW between the diffuse and direct components. We use the direct and diffuse SW data gained from the SPN1 flown during the RACORO campaign to apply a tilt correction for downwelling total SW measurements using Equation 4. We infer the direct normal value directly from the SPN1 measurements by subtracting the measured $D_{T}$ from $G_{T}$, and dividing the result by the calculated $\mu_{\mathrm{T}}$.

\section{Radiometer Offset Characterization}

One factor that affects the ability to apply a tilt correction accurately is the constant offset of the radiometer detector from the level as given by the platform pitch and roll navigation data. These offsets are usually caused by practical limitations on how accurately the radiometers can be mounted level with respect to the platform navigation system readings. Our methodology for determining these constant offsets uses a power law fit to clear-sky measurements with the solar zenith angle as the independent variable as used by Long (JGR, 2000 [14]), and assumes that no significant change in aerosol loading or column water vapor amounts occurs during the data gathering period. For more continuous 
data such as that from ships and buoys, an adaptation of the Long and Ackerman [14] clear-sky identification methodology can be used to detect the clear-sky data needed. But for aircraft campaigns the data are sporadic due to flight length limitations, thus the needed clear-sky data must be provided by a targeted flight. For the RACORO campaign a series of flight patterns was developed in order to determine the instrument pitch and roll offsets. These patterns are similar to those used by other researchers (e.g. Bannehar and Glover [3]) and involve flying multiple headings (typically a box or circular pattern) at a constant altitude and then employing iterative schemes to determine the pitch and roll offsets. The Long and Ackerman clear-sky formula requires clear-sky data over a significant range of solar zenith angles and data close to local solar noon in order to produce the best results for total SW. Thus the RACORO "radiometer offset characterization" flight was aimed at a continuous repeat of the patterns over an entire morning or afternoon under clearsky conditions, and flown at as constant and high an altitude as possible above the boundary layer to minimize the effects of water vapor and aerosol loadings throughout the flight. In the case of RACORO, the flights were flown at a pressure altitude of about 3800 meters. The patterns themselves consisted of a four-leg "box" pattern oriented according to the solar azimuth angle at that time (Fig. 4). The first leg is flown directly along the solar azimuth toward the sun, with the successive three legs flown at right angles to the previous leg. For the "box" pattern the aircraft was held as level as possible or at least as steady as possible given the minimum pitch and roll required to maintain altitude and direction.

Once the above box pattern is completed, the second pattern is a diagonal flight in the direction across the box from corner to corner (gray dashed line in Fig. 4). For this leg, the heading is maintained, and the aircraft flown with various aircraft pitch angles while maintaining a constant and level-as-possible roll angle. The purpose of this leg is to isolate the pitch offsets and effects, holding heading and roll constant. Pitch angles of $+/-2^{\circ}, 5^{\circ}$, and $7^{\circ}$ are flown, and each held for at least 10 seconds in order to allow the CM-22 detector to reach steady state (the CM-22 detector 95\% response time is about 5-7 seconds). For example, the aircraft was flown with the nose pitched down $2^{\circ}$ for $10-15$ seconds, then pitched up $2^{\circ}$ for $10-15$ seconds, then pitched down for $5^{\circ}$ for $10-15$ seconds, etc. This leg was flown as long as needed to complete all 6 pitch angles several times.

In an attempt to gather varying roll angles at zero pitch, a circular third pattern was employed. This third pattern involved holding a constant roll angle at $+/-2^{\circ}, 5^{\circ}$, and $7^{\circ}$ while the aircraft completed a complete $360^{\circ}$ turn for each angle, while maintaining as close to a zero pitch angle as possible. For example, holding the aircraft roll at $2^{\circ}$ for a complete $360^{\circ}$ circle, then holding the aircraft at $-2^{\circ}$ roll for a complete $360^{\circ}$ circle turning in the opposite direction than at $+2^{\circ}$ roll, then holding a $+5^{\circ}$ roll angle for a complete $360^{\circ}$ circle, etc. Unfortunately this flight pattern caused the aircraft to significantly lose altitude during the $360^{\circ}$ circle and, as we will show, the increase in the amount of atmosphere above the aircraft decreased the clear-sky downwelling total SW which significantly precluded its usefulness for inclusion in the clear-sky fit model SW. Subsequent analysis found that since the "box" and "diagonal" patterns are flown for an entire morning or afternoon, the natural change in solar azimuth angle and evolution of winds across this time with the subsequent need to compensate through varying the roll in order to maintain straight flight, sufficient data are obtained of the needed roll angle variations at constant pitch. Thus in the future only the "box" and "diagonal" patterns are needed for aircraft constant pitch and roll offset determination.

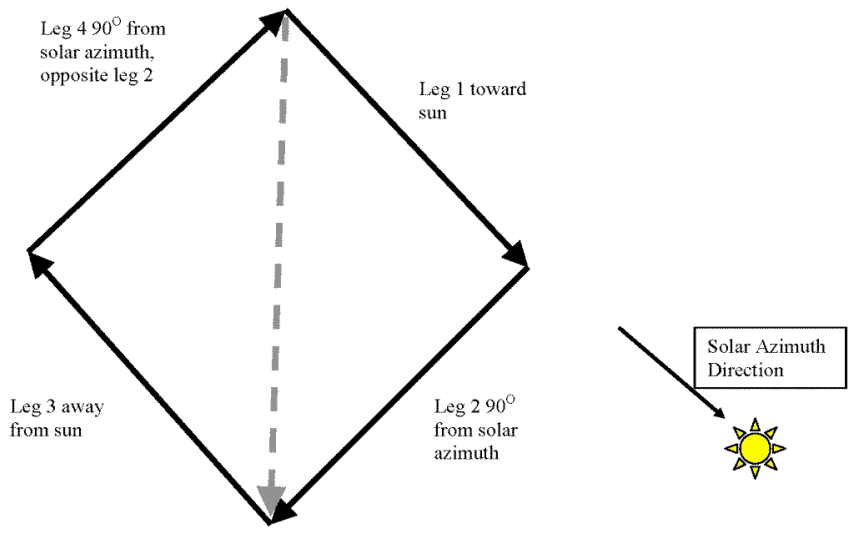

Fig. (4). Illustration of the radiometer offset characterization "box" and "diagonal" patterns used to determine constant pitch and roll offsets of the downwelling SW radiometers.

\section{Pitch and Roll Offset Determination}

Once the radiometer offset characterization flight data is obtained, an iterative process is employed to determine the constant pitch and roll offsets for each radiometer. We use the same methodology of Long and Ackerman [14] for fitting the identified clear-sky flight data, which uses a fitting routine that ignores outliers in the fitting, and uses the cosine of the solar zenith angle $\left(\mu_{0}\right)$ as the independent variable. For each time step, $\mu_{0}$ is calculated using the aircraft navigation data (aircraft latitude, longitude, date, time, etc.). The process steps through varying pitch and roll offsets, calculating $\mu_{\mathrm{T}}$ for each time step and applying a correction based on Equation 4. Once all the "corrected" data for a particular combination of test pitch and roll offsets is produced, the clear-sky fit function is calculated. The iteration proceeds through various values of pitch and roll offsets until the mean absolute deviation of the corrected data from the idealized clear-sky fit is minimized.

Fig. (5) shows the results of the CM-22 pitch and roll offset determination using data from the May 19, 2009 radiometer offset characterization flight. Skies were reported by the pilots to be clear, though at various times during the flight there were some contrails and a small patch of cirrus off in the distance (thus very low in the hemispheric field-ofview of the radiometer). At no time did the isolated contrails or cirrus block the direct sun. The aircraft arrived on station and started the patterns at about 1320 UTC, ending about 1640 UTC (7:20 to $10: 40$ am local time). The time period denoted by the vertical dashed lines in Fig. (5) was used for the tilt offset determination, and maximum tilt for the analysis was limited to $5^{\circ}$ or less. The raw data (light blue) varies by more than $200 \mathrm{Wm}^{-2}$ across the clear-sky fit curve (dark blue), whereas the final tilt corrected data (black) is far better matched to the curve. Note the repeating loss of altitude (dips in the brown trace) during the radiometer offset characterization patterns during this time, which occurs 
during the circular pattern as previously mentioned. The effect (decrease) on the downwelling SW of this decreased altitude can best be seen as the measured versus clear-sky fit difference on the return flight to base at the end of the flight (after about 1640 UTC). The result of the analysis determined that the zenith pointing modified CM-22 radiometer had $\mathrm{a}+1.1^{\circ}$ pitch offset and a $-1.1^{\circ}$ roll offset. The corresponding values for the SPN1 radiometer (not shown) are $+0.9^{\circ}$ pitch and $-1.1^{\circ}$ roll offsets.

Fig. (6) shows the same raw, tilt corrected, and clear-sky fit data as in Fig. (5) used for the tilt offset determination, but with the residuals (tilt corrected data minus clear-sky fit) plotted referenced to the right hand axis. Over these data, the average absolute deviation of the residuals is $4 \mathrm{Wm}^{-2}$, with $95 \%$ of the residuals falling within $+/-10 \mathrm{Wm}^{-2}$ of the clearsky fit across a range of SW spanning from 100 to $1000 \mathrm{Wm}^{-2}$. The residuals, now expanded to include tilt angles up to $+/$ $15^{\circ}$, are plotted in Fig. (7) versus the amount of tilt. As in Fig. (6), the absolute deviation of the residuals is about 4 $\mathrm{Wm}^{-2}$ with $95 \%$ of the residuals within $10 \mathrm{Wm}^{-2}$ for $+/-5^{\circ}$ of tilt. For the range of tilt spanning from $+/-5^{\circ}$ to $+/-10^{\circ}$ (i.e. excluding the $+/-5^{\circ}$ range) the absolute deviation increases to $8.5 \mathrm{Wm}^{-2}$ with $76 \%$ of the residuals within $+/-10 \mathrm{Wm}^{-2}$. For the range of tilt spanning from $+/-10^{\circ}$ to $+/-15^{\circ}$ the absolute deviation increases to $25.5 \mathrm{Wm}^{-2}$ with only $35 \%$ of the residuals within $+/-10 \mathrm{Wm}^{-2}$. Using the residuals for tilt range spanning $+/-10^{\circ}$ inclusive the overall absolute deviation is about $5 \mathrm{Wm}^{-2}$ with $90 \%$ falling within $+/-10$ $\mathrm{Wm}^{-2}$. This result suggests that the tilt correction methodology developed here can reasonably be applied to data with up to $10^{\circ}$ of tilt and still give good results. Beyond $10^{\circ}$ of tilt the data still include significant errors.

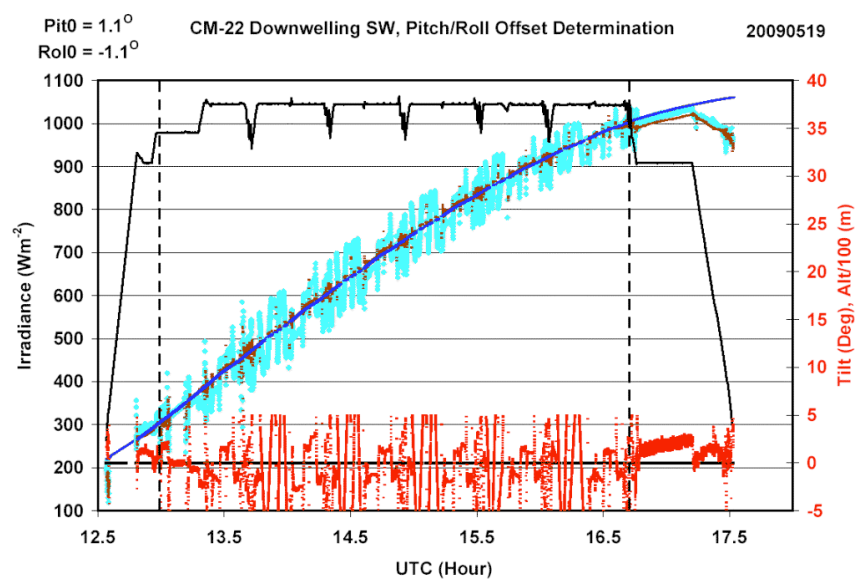

Fig. (5). Data from the May 19 flight showing the raw (light blue), tilt corrected (brown) and clear-sky fit (dark blue) CM-22 data. Red is the amount of instrument tilt from horizontal, black is the aircraft pressure altitude (in $\mathrm{m} / 100$ ) both referenced to the right hand axis. Vertical dashed lines denote the data included in the tilt offset determination.

Another radiometer offset characterization flight had been attempted on May 17, however takeoff was delayed and some cloudiness had developed over the ACRF SGP area, thus the aircraft had to divert 125 miles to the southeast near the Texas/Oklahoma boarder to find clear skies. Fig. (8) shows the radiometer offset characterization data from this May 17 flight, similar to Fig. (6). As one can see, the delay until the aircraft could start the radiometer offset characterization patterns resulted in data being collected over a period roughly centered on local solar noon thus not spanning a sufficient range of solar zenith angles to be useable for tilt offset determination. However these data can be used effectively to test the tilt offset results by applying a tilt correction using the tilt offsets determined from the May 19 data. The residuals across the time span of the characterization pattern show even better agreement than the May 19 data, with an average absolute deviation of only 2 $\mathrm{Wm}^{-2}$ and $99 \%$ of the data falling within $+/-10 \mathrm{Wm}^{-2}$, despite the magnitude of the $\mathrm{SW}$ in this case ranging from 1000 to $1100 \mathrm{Wm}^{-2}$. Pilot reports indicate no clouds at all above the aircraft during these patterns.

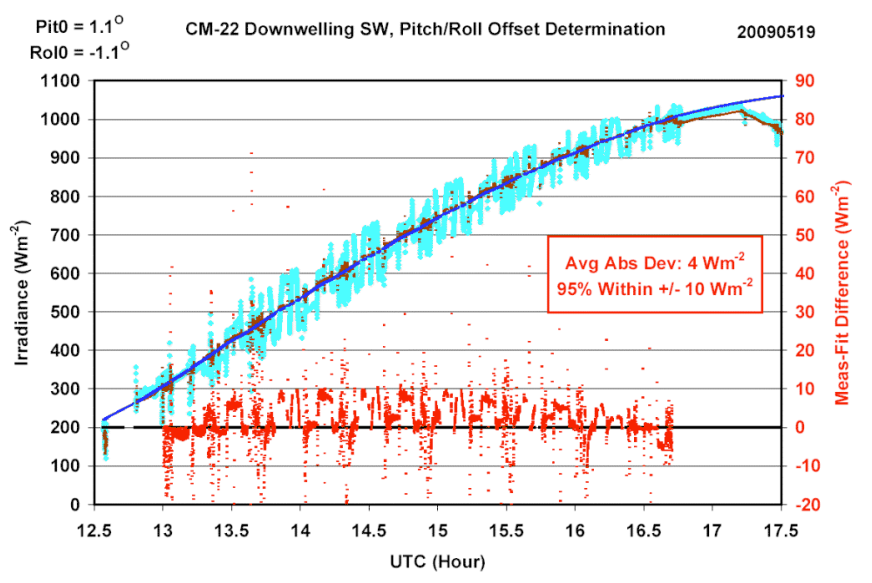

Fig. (6). May 19 flight raw (light blue), tilt corrected (brown) and clear-sky fit (blue) data used for tilt offset determination. Tilt corrected data minus clear-sky fit residuals (red) are referenced to right hand axis.

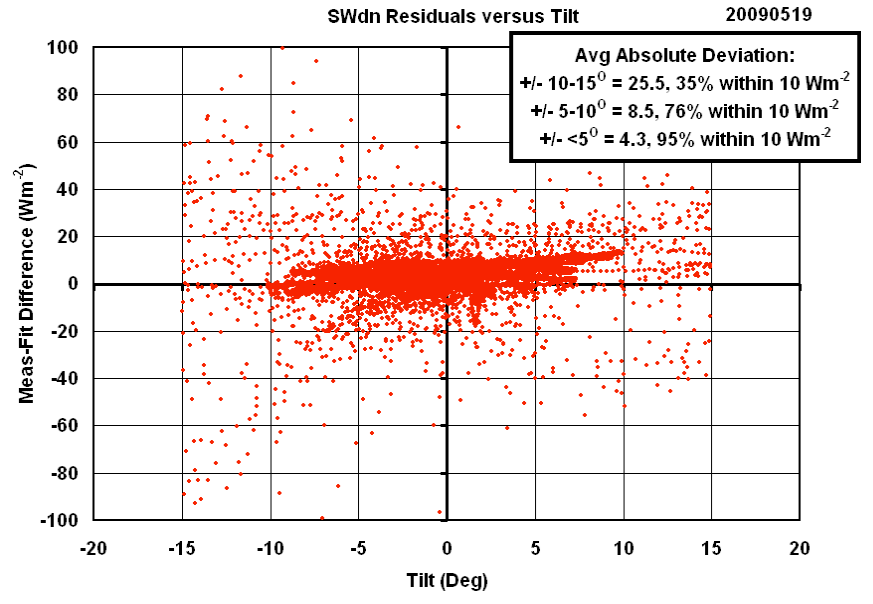

Fig. (7). Residuals of tilt corrected minus clear-sky fit versus tilt for the May 19 flight.

We can also use the May 19th flight data to test the assumption that the diffuse SW is largely unaffected by modest tilt. Fig. (9) top plot shows the raw CM-22 and SPN1 total SW, along with the corresponding SPN1 diffuse SW during the same portion of the flight as used for the offset determination. In this case we include all data up to $15^{\circ}$ of tilt. Note that the total SW fluctuations now span a range of over $400 \mathrm{Wm}^{-2}$ due to the increased tilt occurrences compared to Fig. (5), which was limited to $5^{\circ}$ of tilt. Fig. (9) 
bottom plot shows the diffuse SW plotted as a function of $\mu_{0}$ along with a least-squares linear fit to the data. By looking at the residual difference between the diffuse SW data and the linear fit we, to first approximation, remove the solar zenith angle dependence. Fig. (10) shows the residuals as a function of tilt from horizontal. The correlation between the residuals and tilt is calculated as $0.07,0.12$, and 0.17 for $+/-5^{\circ},+/-$ $10^{\circ}$, and $+/-15^{\circ}$ of tilt inclusive, respectively. While for these three ranges of tilt the correlation is not significant, showing little relationship, the correlation of the wider range of tilt is obviously significantly influenced by the large portion of data for the smaller tilt ranges. Visually, one can see that at least for the $+/-10^{\circ}$ of tilt range, the diffuse residuals are not significantly correlated with tilt, and likely the diffuse variability is caused by other factors including a contribution from inter-detector calibration uncertainty of the SPN1 design as noted previously. Thus, at least for the clearsky case, the assumption that $\mathrm{D} \approx \mathrm{D}_{\mathrm{T}}$ in Equation 3 holds true. It also makes sense that in-cloud or below fairly uniform cloud the diffuse assumption holds, since those situations exhibit about the same level of angular variation in diffuse irradiance as the clear-sky case. How well the diffuse assumption holds for other more angularly varying situations such as below broken cloud remains to be determined.

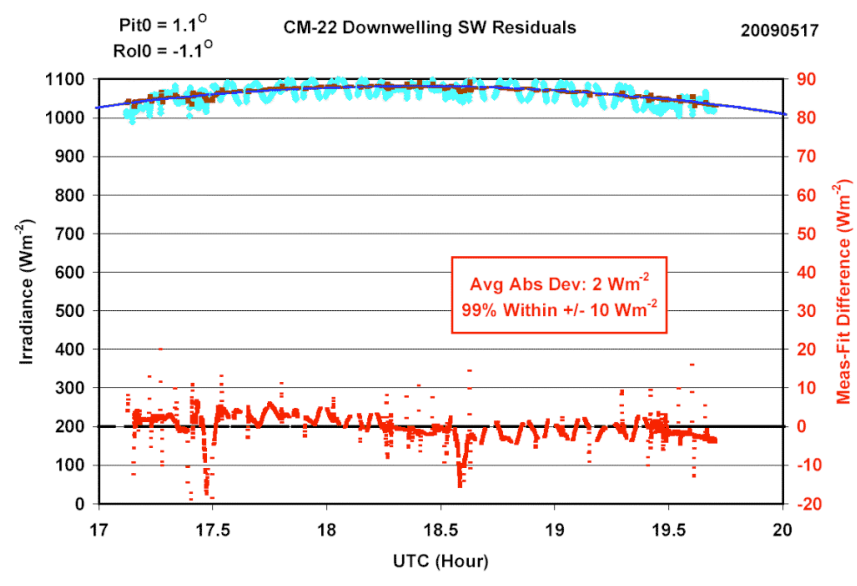

Fig. (8). Same as Fig. (6), but for the May 17 flight.

\section{RESULTS AND STATISTICS FOR RACORO}

Including the radiometer constant tilt offsets to the calculation of $\mu_{\mathrm{T}}$, we apply the tilt correction formula (Equation 4) to all RACORO flight data. To illustrate the effect of either not applying a tilt correction, or applying a "maximum" tilt correction that assumes all downwelling SW is from the direct sun, we look at the May 27 flight. This flight included sampling above the cloud field, descents through the cloud, and sampling below cloud. This flight thus includes some of all three "correction scenarios" of strong direct component, no direct component, and partial direct component with the direct component not completely attenuated by cloud. Fig. (11) shows the raw and tilt corrected CM-22 data, along with the corresponding diffuse SW, and the direct SW. The plot shows the above cloud portions, corresponding to diffuse SW less than $100 \mathrm{Wm}^{-2}$ and significant direct SW, and portions in and below clouds where the direct SW is small to non-existent.
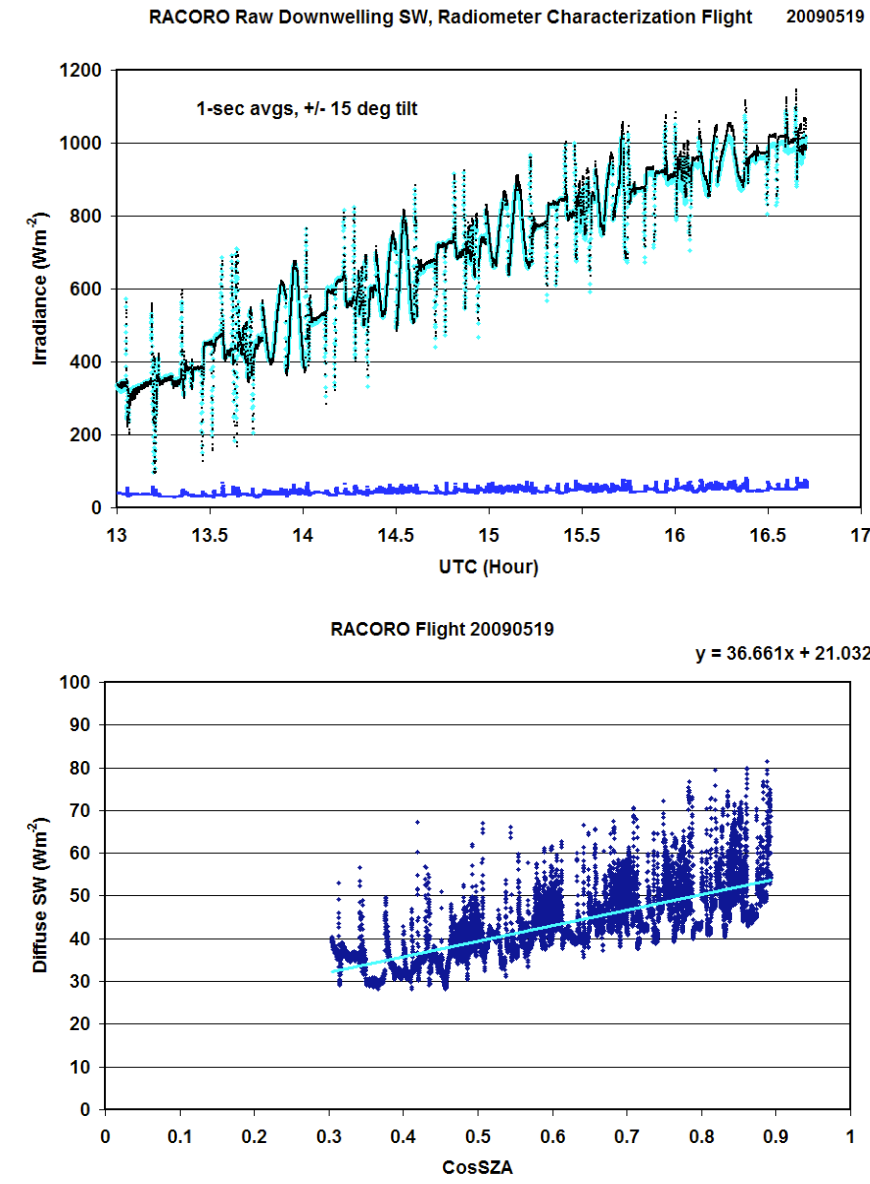

Fig. (9). Top plot - Raw CM-22 (light blue), SPN1 total (black), and diffuse (blue) SW for the tilt offset determination portion of the May 19th flight. Bottom plot - Diffuse SW (blue) versus $\mu_{0}$ along with least-squares linear fit to the data (light blue).

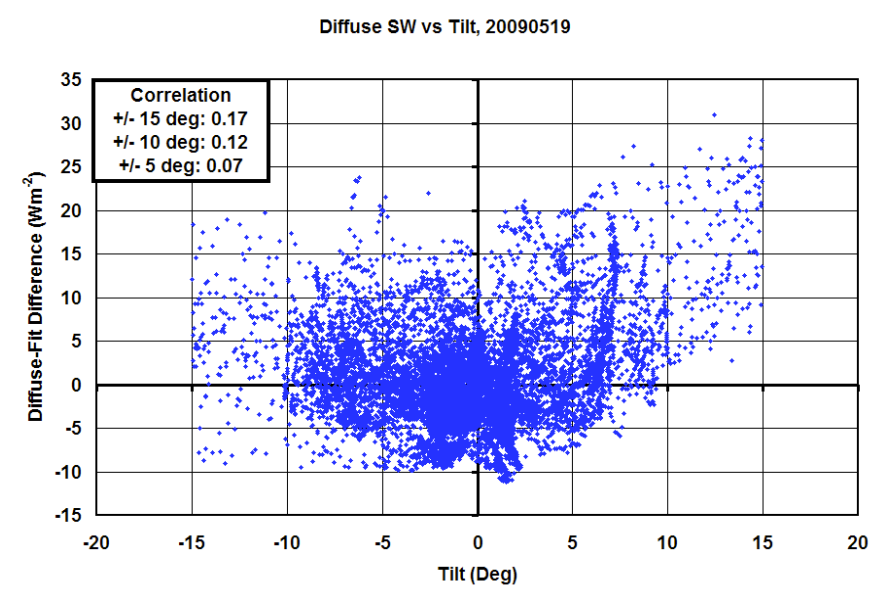

Fig. (10). Diffuse SW minus linear fit residuals versus tilt from the May 19th radiometer offset characterization flight.

For this illustration of tilt correction effects, we limit the data to include only tilt of $5^{\circ}$ or less. (Including a greater range of tilt significantly increases the range of errors.) Fig. (12) shows the difference between not applying any tilt 
correction versus applying a tilt correction using the methodology described here, plotted versus the magnitude of the tilt corrected total SW. Plotting versus the total SW allows for the approximate separation of the differences by the three "correction scenarios" described above, denoted at the top of the plot area in Fig. (12). As expected when there is not a direct component, i.e. the total $\mathrm{SW}$ is comprised only of diffuse SW, there is no difference since in this case no tilt correction should be applied. As the total SW includes more and more direct component, the errors caused by not correcting for tilt become larger, in the May 27 case errors of up to $40 \mathrm{Wm}^{-2}$ for tilt of $5^{\circ}$ or less.

Downwelling SW, May 27 Clear/Cloud case

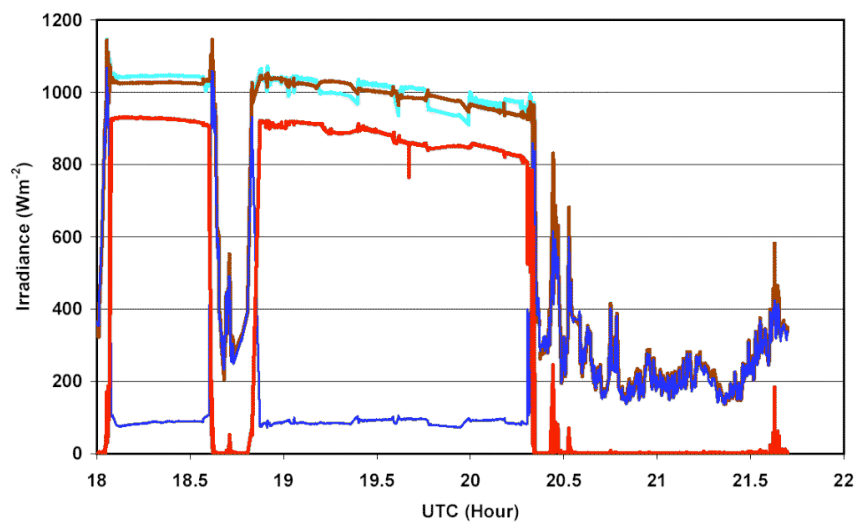

Fig. (11). Raw (light blue), tilt corrected (brown), diffuse (blue) and direct (red) SW data from the May 27 flight.

Fig. (13) shows the differences from our tilt correction and applying the "maximum" correction which assumes all direct SW. In this case errors are exhibited for all three "correction scenarios" again of magnitude of up to $40 \mathrm{Wm}^{-2}$. For the thick overcast (i.e. no direct component) case on May 27 the error becomes a significant fractional proportion of the lower magnitude total SW, up to about $10 \%$ for 40 $\mathrm{Wm}^{-2}$ of error out of $400 \mathrm{Wm}^{-2}$ total SW. Even in the clearsky case, assuming all direct $\mathrm{SW}$ produces significant errors (up to $20 \mathrm{Wm}^{-2}$ ) since there is in this case just less than 100 $\mathrm{Wm}^{-2}$ of diffuse SW (Fig. 11). Errors of these magnitudes will significantly impact scientific studies such as the measurement of radiative heating rate profiles where the flux divergence is most often the small difference between large irradiance values. Errors of the magnitude shown here are often about the same magnitude as the flux divergence being sought, precluding accurate determination of layer heating rates. We conclude that more properly correcting for tilt from horizontal in these type data, when the benefits of a stabilized platform are not available, is essential for studies requiring a high degree of accuracy.

As noted previously, the RACORO campaign included 259 research flight hours during 59 flights. While the primary purpose of RACORO was to sample boundary layer liquid-water cloud fields, the abundance of flight hours budgeted for the campaign allowed for other scientifically useful sampling as well, including surface spectral albedo mapping flights, boundary layer turbulence sampling flights, and aerosol variability sampling flights. These latter flights were flown when RACORO cloud conditions were not present, and most often included conditions where the direct sun was not significantly attenuated by intervening cloud.
Thus a major portion of the overall RACORO flight time included conditions where significant errors occur if a tilt correction that includes knowledge of the direct and diffuse SW partitioning is not applied, as illustrated in Figs. $(\mathbf{1 2}, \mathbf{1 3})$.

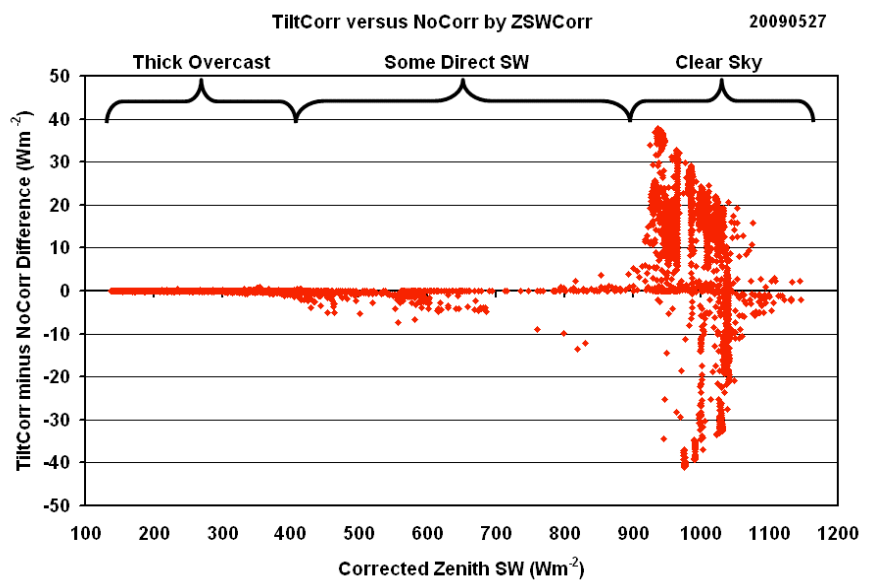

Fig. (12). Difference between our tilt corrected and no correction applied versus total corrected SW for the May 27 flight for up to $5^{\circ}$ of tilt.

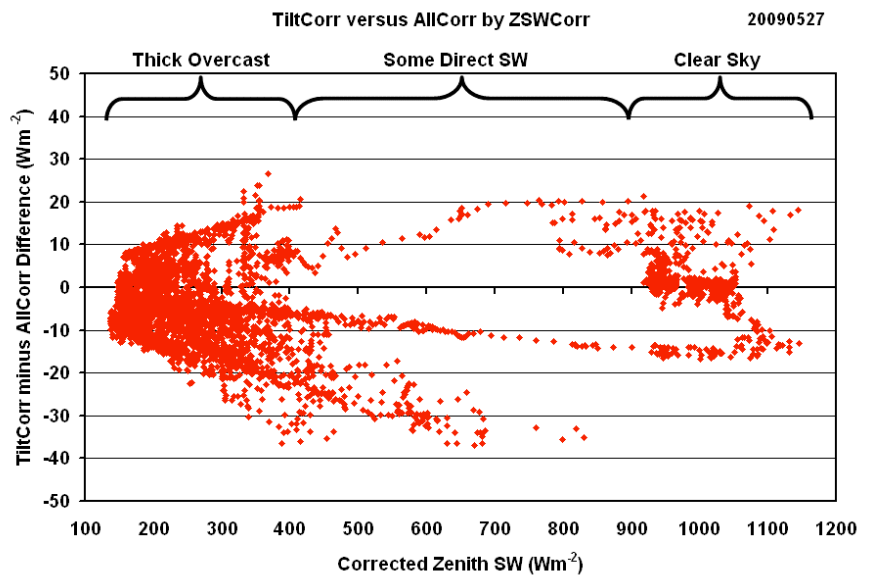

Fig. (13). Difference between our tilt corrected and maximum correction applied versus total corrected SW for the May 27 flight for up to $5^{\circ}$ of tilt.

Fig. (14) shows the frequency distribution of the amount of tilt that was present in the aggregate for all RACORO flight hours, including takeoff, landing, transits to the experiment area, and sampling. The largest frequency in the bi-modal distribution occurs for $3-5^{\circ}$ of tilt. This is influenced by the nature of aircraft flight where typically about $3^{\circ}$ of pitch is needed to maintain flight altitude. Analysis of the cumulative frequency of occurrence (Fig. 15) shows that only about $4 \%$ of the flight time had tilt greater that $10^{\circ}$, but about $25 \%$ of the time had tilt greater than $5^{\circ}$. Thus if a maximum of $5^{\circ}$ tilt for correctable data is used, about $25 \%$ of the RACORO radiation data would be removed from the total RACORO sampling. On the other hand, given our analysis here that suggests good tilt correction results for up to $10^{\circ}$ of tilt, only a small percent of the RACORO radiation data is deemed unusable.

Fig. (16) shows the frequency distribution of the correction factors (the factor that $\mathrm{G}_{\mathrm{T}}$ is multiplied by, i.e. the 
term in parentheses in Equation 4) applied to the RACORO data. $93 \%$ of the data had correction factors of $+/-0.1$ from unity applied. In other words, the total measured SW was adjusted by an amount of $10 \%$ or less. $73 \%$ of the time, the total SW was adjusted by $5 \%$ or less. Another way of viewing this result is that $27 \%$ and $7 \%$ of the time the tilt from horizontal produced errors greater than $5 \%$ or $10 \%$ of the measured amount, respectively.

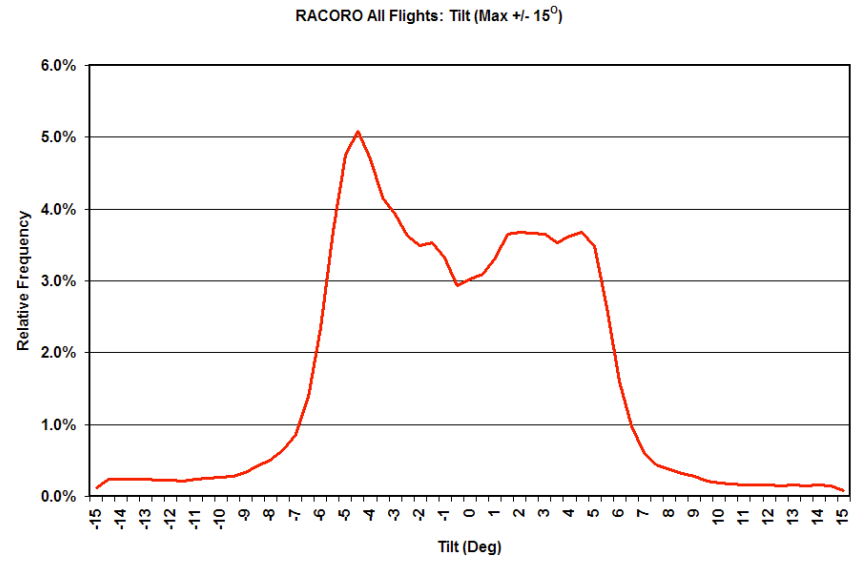

Fig. (14). Frequency of occurrence for the amount of tilt encountered during the RACORO campaign including all flights.

RACORO All Flights: Tilt $\left(\operatorname{Max}+/-15^{\circ}\right)$

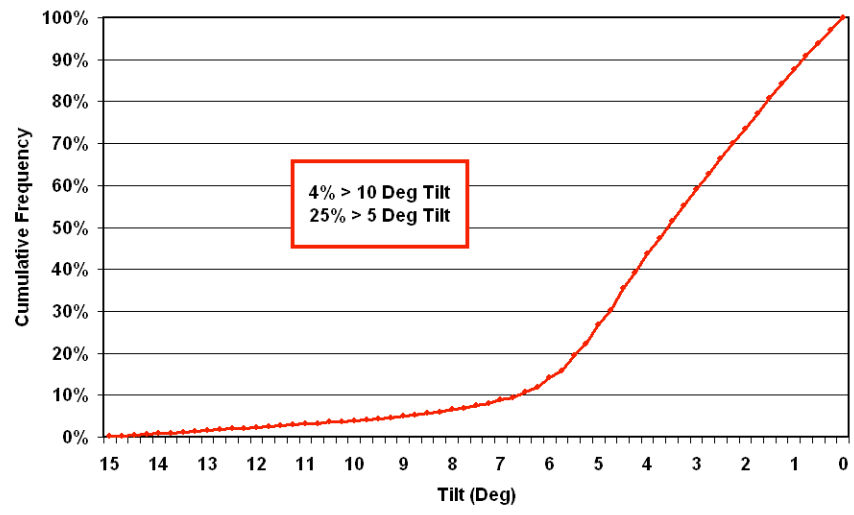

Fig. (15). Cumulative frequency of occurrence for the amount of tilt encountered during the RACORO campaign including all flights.

The actual amount of adjustment in terms of irradiance units are shown in Fig. (17). The blue line represents all data, and shows that $42 \%$ of the time the amount of tilt correction applied is $15 \mathrm{Wm}^{-2}$ or less. At the same time, $20 \%$ of the data required a tilt correction of $45 \mathrm{Wm}^{-2}$ or greater. This distribution to first order mimics the distribution of correction factor shown in Fig. (16). We screen the data for times when there is a significant direct component for the frequency of occurrence shown in red, which represents all times when the direct normal irradiance was $500 \mathrm{Wm}^{-2}$ or greater, and corresponds to the "some direct SW" and "clearsky" scenarios described in Figs. $(\mathbf{1 2}, \mathbf{1 3})$. It is not surprising given the boundary layer clouds targeted by the RACORO campaign which are often broken or tenuous, plus the other non-cloud-targeted scientific flights, that there occurred a significant direct component in $76 \%$ of the flight data

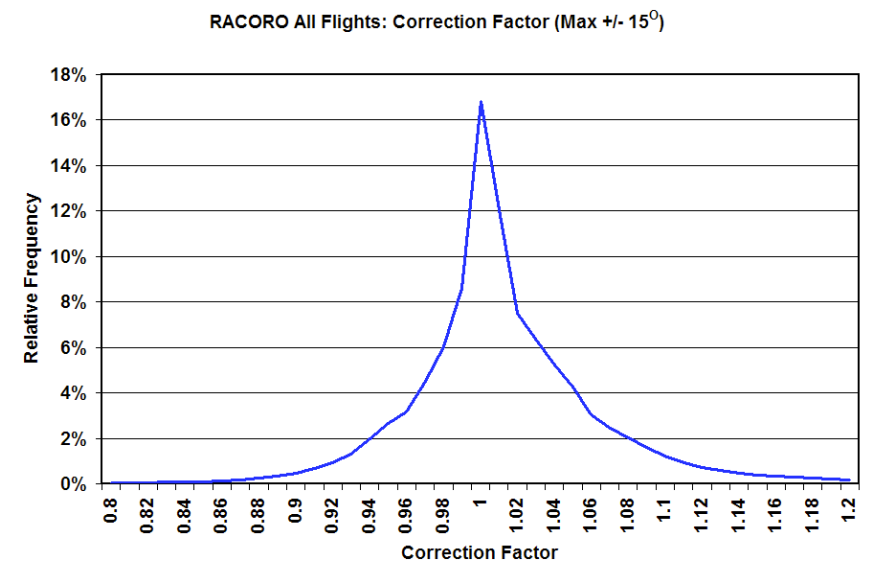

Fig. (16). Frequency of occurrence of the tilt correction factor applied to the raw measured $\mathrm{CM}-22$ data during the RACORO campaign including all flights.

collected. In these cases, the distribution more resembles the bimodal frequency of occurrence of amount of tilt shown in Fig. (14). When a significant direct component is present, only $27 \%$ of the time the amount of tilt correction applied is $15 \mathrm{Wm}^{-2}$ or less, and $26 \%$ of the data required a tilt correction of $45 \mathrm{Wm}^{-2}$ or greater. The remainder of the time $(48 \%)$ a tilt correction of between 15 and $45 \mathrm{Wm}^{-2}$ is needed. This once again serves to illustrate the importance of knowing the partitioning between the direct and diffuse SW components in order to better account for tilt from horizontal.

\section{SUMMARY}

We have shown that, unless corrected, significant errors occur in downwelling SW measurements made on moving platforms due to tilt from horizontal using data from aircraft flights during the RACORO field campaign. A-priori knowledge of the partitioning between the direct and diffuse components of the total SW is needed to properly apply a correction for tilt. This partitioning information can be adequately provided using a newly available commercial radiometer, the SPN1, that produces reasonable measurements of the total and diffuse SW (and by subtraction the direct SW) with no moving parts and regardless of azimuthal orientation. We have developed methodologies for determining the constant pitch and roll offsets of the radiometers for aircraft applications, and for applying a tilt correction to the total SW data. Results suggest that the methodology is accurate to better than 5 $\mathrm{Wm}^{-2}$ absolute deviation for tilt up to $+/-10^{\circ}$, with $90 \%$ of the data corrected to within $10 \mathrm{Wm}^{-2}$ at least for the clear-sky data analyzed here. Without a proper tilt correction, even data limited to $5^{\circ}$ of tilt still exhibits large errors, greater than $100 \mathrm{Wm}^{-2}$ in some cases. Given the low cost, low weight, and low power consumption of the SPN1 total and diffuse radiometer, opportunities previously excluded for moving platform measurements such as small Unmanned Aerial Vehicles and solar powered buoys now become feasible. The increase in measurement accuracy is important, given current concerns over long-term climate variability and change especially over the $70 \%$ of the Earth's surface covered by ocean where long-term records of these 
measurements are sorely needed and must be made on ships and buoys.

RACORO All Flights: Correction Amount (Max $\left.+/-15^{\circ}\right)$

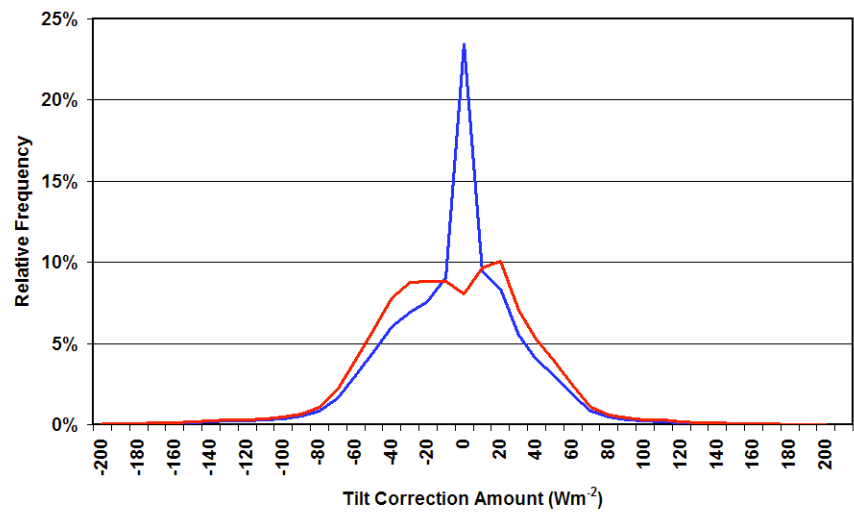

Fig. (17). Frequency of occurrence of the tilt correction amount applied to the raw measured CM-22 data during the RACORO campaign including all data (blue) and times when at least $500 \mathrm{Wm}^{-2}$ of direct normal SW was present (red).

Further work is needed to quantify the accuracy of the tilt correction methodology presented here, including testing of the equivalent diffuse assumption for modest tilt under partly cloudy skies, and comparison to actual accurate measurements rather than only clear-sky fitted functions. We recommend a dedicated campaign where a set of SW radiometers are mounted on the frame as in the RACORO campaign and an equivalent set mounted on a properly working stabilized platform where a tilt correction should not be needed. Data for all three "correction scenarios" as denoted in Figs. $(\mathbf{1 2}, \mathbf{1 3})$ need to be well sampled to produce a statistically sufficient data set for quantifying the ability of our methodology to accurately correct for tilt from horizontal under various types of cloudiness conditions.

\section{ACKNOWLEDGEMENT}

The authors acknowledge the support of the Climate Change Research Division of the U.S. Department of Energy as part of the ARM Climate Research Facilities Program. Recognition is also extended to those responsible for the operation and maintenance of the aircraft instruments that produced the data used in this study; their diligent and dedicated efforts are often underappreciated.

\section{REFERENCES}

[1] Bucholtz A, Bluth RT, Kelly B, et al. The stabilized radiometer platform (STRAP) - An actively stabilized horizontally level platform for improved aircraft irradiance measurements. J Atmos Oceanic Technol 2008; 25: DOI: 10.1175/2008JTECHA1085.1.

[2] Wendisch M, Muller D, Schell D, Heintzenberg J. An airborne spectral albedometer with active horizontal stabilization. J Atmos Oceanic Technol 2001; 18: 1856-66.

[3] Bannehar L, Glover V. Preprocessing of airborne pyranometer data, NCAR Tech 1991; pp. 35. Note NCAR/TN-364+STR

[4] Saunders RW, Brogniez G, Buriez JC, Meerkotter R, Wendling p. A comparison of measured and modeled broadband fluxes from aircraft data during the ICE ' 89 field experiment. J Atmos Oceanic Technol 1992; 9: 391-406.

[5] Boers R, Mitchell RM, Krummel PB. Correction of aircraft pyranometer measurements for diffuse radiance and alignment errors. J Geophys Res 1998; 103: 16753-8.

[6] McDowall G. Broadband solar irradiances measured on fixed and stabilized platforms: comparison of observations and their uncertainties, Masters Thesis, Florida State Univ 2005; p. 40.

[7] Vogelmann AM, McFarquhar G, Ogren j, et al. "RACORO Science and Operations Plan," DOE/SC-ARM-0806, December 2008, 39 pgs. (http://www.arm.gov/publications/programdocs/doesc-arm-0806.pdf)

[8] Guan H, Schmid B, Bucholtz A, Bergstrom R. The sensitivity of shortwave radiative fluxes, forcing, and heating rates to the aerosol vertical profile. J Geophys Res 2009; in press.

[9] Bucholtz A, Jonsson H. Modified Kipp \& Zonen pyranometers and pyrgeometers for use on atmospheric research aircraft. J Atmos Oceanic Technol [in preparation for submission to].

[10] Kipp \& Zonen (2003), CM-22 pyranometer instruction manual. Kipp \& Zonen, pp. 61 [Available on-line at http://www.kippzonen. com/?downloadcategory/991/Discontinued+Products.aspx]

[11] ASTM International (2005), Standard Test Method for Calibration of a Pyranometer Using a Pyrheliometer, ASTM G 167-05, p. 21.

[12] Hickey JR, Daniels DB, Nelson DW. Automated (HF) cavity pyrheliometer systems: Operational Experience, Test Results and Modification, Proceedings of the 1993 Annual Conference American Solar Energy Society, Washington, D. C., April 25-28, 1993.

[13] Moon P, Spencer DE. Illumination from a non-uniform sky.Trans Illum Eng Soc NY 1942; 37: 707.

[14] Long CN, Ackerman TP. Identification of clear skies from broadband pyranometer measurements and calculation of downwelling shortwave cloud effects, JGR, 2000; 105: No. D12, 15609-26. 